\title{
3D NON-INVASIVE INSPECTION OF THE SKIN LESIONS BY CLOSE- RANGE AND LOW-COST PHOTOGRAMMETRIC TECHNIQUES
}

\author{
AHMET B. ORUN ${ }^{凶, 1}$, ERIC GOODYER ${ }^{1}$, GEOFF SMITH $^{2}$ \\ ${ }^{1}$ De Montfort University, Faculty of Technology, Gateway House. Leicester LE1 9BH, UK; ${ }^{2}$ De Montfort \\ University, Faculty of Health and Life Sciences, Leicester, UK Ahmet \\ e-mail: aorun@dmu.ac.uk,eg@dmu.ac.uk,gsmith02@dmu.ac.uk \\ (Received March 24, 2017; revised November 27, 2017; accepted November 27, 2017)
}

\begin{abstract}
In dermatology, one of the most common causes of skin abnormality is an unusual change in skin lesion structure which may exhibit very subtle physical deformation of its 3D shape. However the geometrical sensitivity of current cost-effective inspection and measurement methods may not be sufficient to detect such small progressive changes in skin lesion structure at micro-scale. Our proposed method could provide a low-cost, non-invasive solution by a compact system solution to overcome these shortcomings by using close-range photogrammetric imaging techniques to build a 3D surface model for a continuous observation of subtle changes in skin lesions and other features.
\end{abstract}

Keywords: bundle adjustment, low-cost inspection, skin imaging, stereoscopy.

\section{INTRODUCTION}

For dermatological diagnosis, the detection of very early signs of disease, whether physical or structural, is important to ensure early intervention. The measurement sensitivity of current methods may not be sufficient to detect these early signs of disease (melanoma, etc.), which present as changes on the skin features. Small physical changes can be efficiently detected in the $3 \mathrm{D}$ domain by using photogrammetric measurement techniques at the required accuracy. Our proposed method is a low-cost non-invasive solution that overcomes the accuracy problems. This is achieved by using a micro-scale photogrammetric bundle adjustment method (Granshaw, 1980) for all 3D skin features, including skin lesions or wounds. The photogrammetric bundle adjustment technique has been widely used in industrial applications (Orun and Alkis, 2003) and aerial photography (Orun and Natarajan, 1994) since 1950, but now its compactportable version may also be utilized at micro scale for medical diagnostic purposes by observing the subtle progressive changes in a lesion structure, or any other physical skin feature (volume, shape, etc.). The techniques proposed within this research can also be applicable to subcutaneous layers by utilizing a high resolution $I R$ camera to detect micro-level progressive changes of skin features over a specific time period. Nowadays most skin imaging techniques are limited to the 2D domain, and look for the skin fea- tures such as color change, lesion symmetry, lesion border irregularity check, etc. (Claridge and Orun, 2002; 2003) and are unable to make accurate progressive $3 \mathrm{D}$ observations of skin abnormalities such as malignant lesion growth, displacement of micro blood vessels or suspicious changes in mole at micro scale. Other studies which focus on medical 3D feature reconstruction also have some disadvantages. Alvarez et al. (2006) investigated the use of digital photography to measure wounds. They developed the Photo-Digital Planimetry Software (PDPS) utility for this purpose but limited it to $2 \mathrm{D}$ planimetric measurements. One other work similar to ours was introduced by Gorpas et al. (2007). They use high-cost twin CCD cameras with telecentric lenses for three-dimensional lesion surface measurements. The cameras they utilized have to be fixed and carefully located; which results in many restrictions to the image data collection process, hence this method is not practical for a flexible clinical environment. In contrast our system uses a low-cost single camera (40-50 times cheaper than the telecentric lenses used by Gorpas et al., 2007) with a completely free-hand image acquisition style without the need for a fixed camera position or any geometric setup restriction. For medical applications some researchers have used 3D object reconstruction techniques. Choi et al. (2013) used a comprehensive photogrammetrical system with 3 digital cameras for orthognatic surgery. Goellner et al. (2010) also introduced a similar system used in dentistry which utilizes stereo camera pairs with photo- 
grammetric technique. Such systems can only be used with a highly conditioned setup in a restricted environment and are not cost effective. With regards to low-cost solutions, one of studies introduces a close range photogrammetric system using a single camera which was conducted by Khalil (2011). In his experiments he measures the displacement of a moving object only in $\mathrm{X}, \mathrm{Y}$ directions by keeping the camera at fixed position and the object $Z$ coordinates are disregarded. The theory of photogrammetrical measurement precision by using single camera was studied by Luhman (2009) comprehensively. He emphasizes the importance of avoiding photogrammetrical system restrictions (e.g., synchronization of cameras, spatial observation conditions, system costs, etc.). The novelty used within this work is a specifically designed "reference ring" by which an ordinary (low cost, nonmetrical) camera can be used with free-hand (nonfixed stationary) image acquisition. This characteristics would also lead to more frequently self-inspection of suspicious skin moles by a non-expert user. This is because of simple usage of the system by a simple stereo image collection without a complicated instrumentation setup. Such system portability and simplicity (e.g., by single camera in a mobile phone) also allows quantitative measurements of a suspicious mole to be transmitted to clinical domain for an instant analysis.

\section{MATERIAL AND METHODS}

\section{PHOTOGRAMMETRIC IMAGE ACQUISITION}

Photogrammetry is a fundamental technique which specifies the geometrical relationship between image points (any point of an object whose image is taken) and the three dimensional Cartesian coordinates of an object (e.g., skin lesion). Each point in the scene is represented by a unique feature such as an edge, dot or tip of a line, etc. and must also be identified in the corresponding stereo images of the scene. Nowadays many photogrammetric methods are used in several fields of medical imaging but in this study we focus on medical close-range photogrammetry, and particularly the bundle adjustment method, which is sub-set of photogrammetry (Moffitt and Mikhail, 1980). A simple stereoscopic image acquisition process in association with the bundle adjustment technique can be achieved (Fig. 1) by using a low-cost single digital camera, and by taking a pair of images of a skin lesion from two different positions $\left(\mathrm{S}_{1}\right.$ and $\left.\mathrm{S}_{2}\right)$.In the experiments a reference ring is used whose markings were already measured accurately, to provide Cartesian coordinates of Control and Check Points, as shown in Fig. 2. The rings rear side is coated with an adhesive material so that it can be vertically positioned on the surface surrounding a skin lesion. Left and right stereoscopic images of the skin lesion are used to create 3D lesion modelling. The Accuracy of the control points $(C P)$ marked by tiny holes on the reference ring, directly effect the accuracy of the results yielded by the bundle adjustment algorithm. The check points located at the tip of test bars (Fig. 2) whose X,Y,Z object coordinates are also known enable corrections to be made to the final lesion model coordinates.

\section{BUNDLE ADJUSTMENT TECHNIQUE}

The photogrammetric bundle adjustment technique used here aims to generate 3D skin lesion models at micro scale. A similar technique was previously used by Jaspers et al. (1999) by a micro-mirror device instead of a single digital camera. There are also various applications in which close-range photogrammetric principles used. Unlike conventional closerange photogrammetry, our suggested application has compact-portable characteristics used with a bundle adjustment utility. The basic method was previously introduced by the authors such as Orun and Natarajan, 1990; and also more earlier introduced by Granshaw, 1980. A brief description of bundle adjustment may be explained as follows. In Fig. 1,

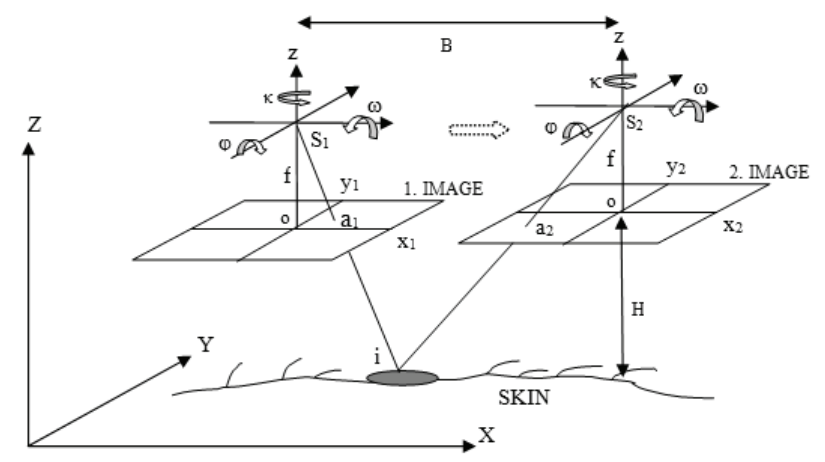

Fig. 1. Stereoscopic image acquisition by non-fixed stationary photogrammetric technique using a single camera whose center of locations are depicted as $S_{I}$ and $S_{2}$. Calculation of a single point (i) coordinates on a skin lesion is made by bundle adjustment iteration algorithm to build $3 D$ model. In the Figure, base (B) refers to distance between the $S 1$ and $S 2$, height $(H)$ is the distance between the camera position $\left(S_{i}\right)$ and skin surface. 

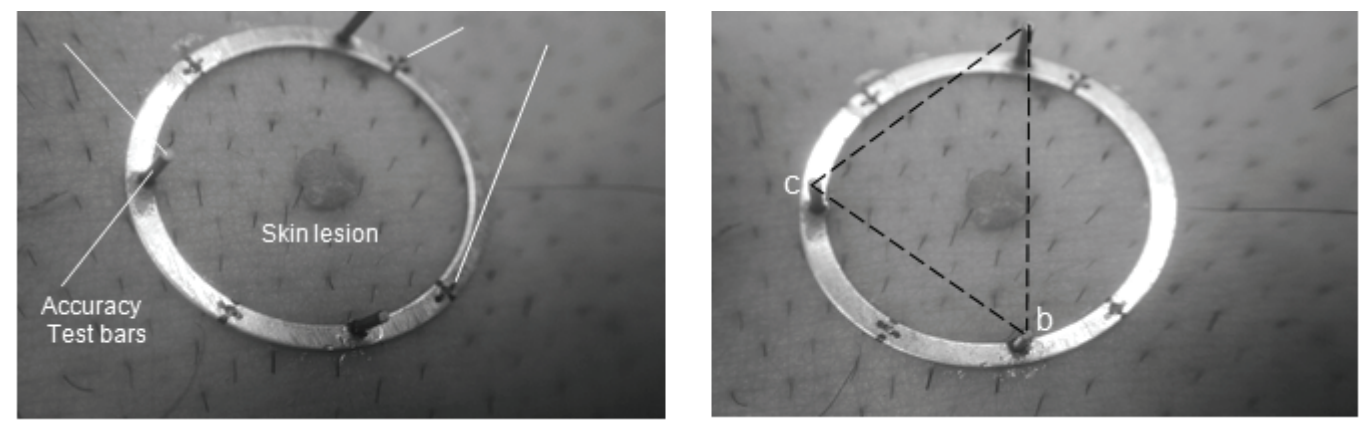

Fig. 2. Left and right stereoscopic images of a skin lesion are acquired for 3D lesion modelling. The accuracy of control points on the reference ring (marked as " + ") directly effect the results yielded by the bundle adjustment algorithm. Meanwhile the check points located at the tip of test bars are used to correction the lesion model coordinates. The triangle area (at the right image) is also used for interpolation of the check point coordinate values to correct the lesion coordinates.

We can consider $i_{t h}$ point on the skin surface with the spatial co-ordinates of $(\mathrm{X}, \mathrm{Y}, \mathrm{Z})_{\mathrm{i}}$ as it corresponds to pair of image points $\mathrm{a}_{1}$ and $\mathrm{a}_{2}$, and whose image coordinates are $\left(\mathrm{x}_{\mathrm{i}}, \mathrm{y}_{\mathrm{i}}\right)_{1,2}$ on (stereoscopic) image sequences 1 and 2. In Fig. 1 and Eq. 1, $\mathrm{f}$ is the principal distance (or focal length if $f_{\text {objective }}=\infty$ ), which are already calculated by a basic camera calibration in the lab conditions (Section 2.3). The principal distance (focal length) is a camera interior parameter and has to be re-calculated after the modification of the camera lens system (e.g. attaching a close range objective), which makes the camera suitable for nonfixed (free-hand) stationary measurements. (X, Y, $\mathrm{Z})_{\mathrm{S} 1, \mathrm{~S} 2}$ are the cameras' perspective centers (camera locations) coordinates and $(\mathrm{w}, \varphi, \kappa)_{1,2}$ refers to the camera rotation angles for locations 1 and 2 . The collinearity Eq. 1 define the relationship between the image coordinates and the surface coordinates of the same skin lesion points.

$x_{i}=-f \frac{r_{11}\left(X_{i}-X_{s}\right)+r_{21}\left(Y_{i}-Y_{s}\right)+r_{31}\left(Z_{i}-Z_{s}\right)}{r_{13}\left(X_{i}-X_{s}\right)+r_{23}\left(Y_{i}-Y_{s}\right)+r_{33}\left(Z_{i}-Z_{s}\right)}$

$y_{i}=-f \frac{r_{12}\left(X_{i}-X_{s}\right)+r_{22}\left(Y_{i}-Y_{s}\right)+r_{32}\left(Z_{i}-Z_{s}\right)}{r_{13}\left(X_{i}-X_{s}\right)+r_{23}\left(Y_{i}-Y_{s}\right)+r_{33}\left(Z_{i}-Z_{s}\right)}$

In the equations $r_{i j}$ are the elements of the image rotation matrix for the each image. These collinearity equations are solved by iteration method to calculate the $\mathrm{X}, \mathrm{Y}, \mathrm{Z}$ object coordinates. This solution can only be possible by an inverse solution algorithm, this is because the majority of parameters are unknown, and only the image co-ordinates $\left(\mathrm{x}_{\mathrm{i}}, \mathrm{y}_{\mathrm{i}}\right)_{1,2}$ can be measured on the pair of stereo image pairs. In Eq. 1, the $r_{i i}$ elements belong the rotation matrix $R$ which is an orthogonal matrix, that is a basic feature in photogrammetry. The bundle adjustment technique uses an inverse solution of the collinearity equations (1) by iteration method. Before this process, the collinearity equations have to be linearized by Taylor's Theorem, and then the normal equations can be established from these linearized observation equations. If we define the partitioned normal equations (Granshaw, 1980) as below :

$$
\left(\begin{array}{cc}
N_{p} & N_{p s}^{T} \\
N_{p s} & N_{s}
\end{array}\right)\left(\begin{array}{l}
\Delta x_{p} \\
\Delta x_{s}
\end{array}\right)=\left(\begin{array}{l}
t_{p} \\
t_{s}
\end{array}\right)
$$

In the Eq. 2, $p$ denotes surface point co-ordinates and $s$ refers to the camera parameters, $N_{\mathrm{ps}}$ denotes the normal equations for them. In the equation, $\Delta x_{p}$ and $\Delta \mathrm{x}_{\mathrm{s}}$ denote the corrections to each surface point coordinates $(X, Y, Z)_{i}$ and also the camera parameters $\left(X_{s}\right.$ $\left., Y_{s}, Z_{s}, \omega, \varphi, \kappa\right)$ respectively. Here $\mathrm{t}_{\mathrm{p}}$ and $\mathrm{t}_{\mathrm{s}}$ are the corresponding right hand sides of normal equations. Based on Eq. 2, the inverse form of sub-matrix $\mathrm{N}_{\mathrm{p}}$ can be calculated, which then form the reduced normal equations:

$$
\begin{array}{ll} 
& \mathrm{N}_{\mathrm{r}} \Delta \mathrm{x}_{\mathrm{s}}=\mathrm{t}_{\mathrm{r}}, \\
\text { where : } & N_{r}=N_{s}-N_{p s} N_{p}^{-1} N_{p s}^{T}, \\
\text { and } \quad & t_{r}=t_{s}-N_{p s} N_{p}^{-1} t_{p},
\end{array}
$$

$\Delta \mathrm{x}_{\mathrm{s}}$, and $\Delta \mathrm{x}_{\mathrm{p}}$ can be calculated from the Equation 3 as follows;

$$
\Delta x_{p}=N_{p}^{-1}\left(t_{p}-N_{p s}^{T} \Delta x_{s}\right),
$$

$\Delta \mathrm{x}_{\mathrm{p}}$ is referred to $(\delta \mathrm{X}, \delta \mathrm{Y}, \delta \mathrm{Z})_{\mathrm{i}}$ which denote the corrections for each surface point's co-ordinates. The values above can be calculated by iteration method. It takes a few iterations in our system to reach the solution depending on the initial approximate values. At least four points' co-ordinates should be included into the normal equations. 


\section{BASIC CAMERA CALIBRATION PROCESS (CALCULATION OF PRINCIPLE DISTANCE)}

The principal distance (or focal length if $f_{\text {objective }}=\infty$ ) can be calculated by a basic level of camera calibration process in a lab environment (Fig. 3). This process has to be done after the modification of the camera lens system to make the camera suitable for non-fixed stationary measurements. The focal length $(f)$ is a camera interior parameter and can be calculated as (After M.Hiraoglu, 1992);

$$
f_{m m}=\frac{\Delta L_{m m}}{\left(d_{m m} /\left(S_{1} \cdot P x_{m m}\right)\right)-\left(d_{m m} /\left(S_{2} \cdot P x_{m m}\right)\right)},
$$

$\mathrm{d}$ : A measured distance between two dots (in the dotgrid calibration plate)

Px: Single CCD detector size of the camera (e.g., $3.1 \times 3.1$ micron)

$\Delta \mathrm{L}$ : Distance between the two positions of calibration plate

$\mathrm{S}_{1}, \mathrm{~S}_{2}$ : length of "s" measured on the image (in pixel unit) for positions 1 and 2 .

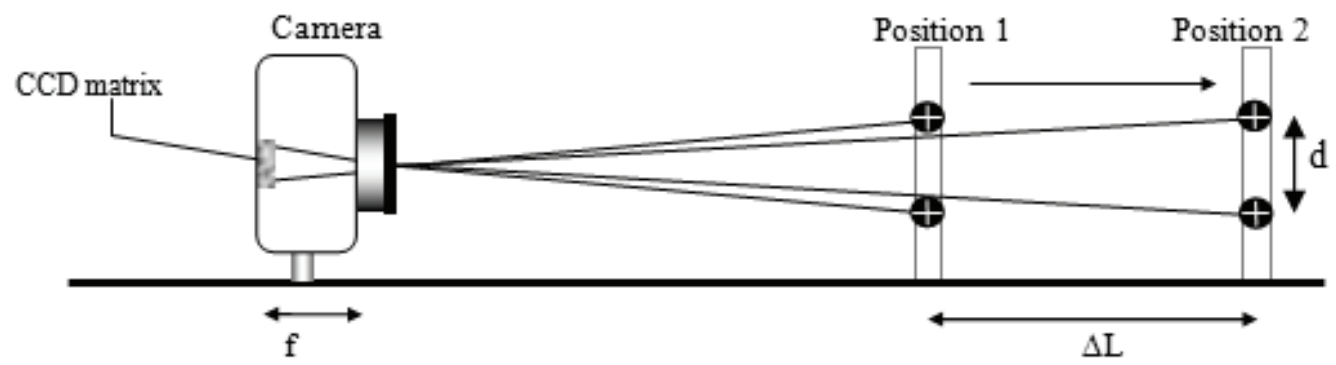

Fig. 3. The calibration utility configuration to define camera focal length (principal distance). The images are taken at two different positions ( 1 and 2) of dots-grid calibration plate.

In our experiments a low cost (less than $\$ 100)$ digital camera (Premier, KSI Trade ltd, UK) with $2032 \times 1520$ pixel resolution is used for the image acquisition. The methods introduced within this study require a digital $C C D$ camera having a resolution of at least $2000 \times 1500$ pixels with non-auto focused lens and non-auto zoom characteristics.

\section{RESULTS}

Experimental tests have been accomplished to investigate how the bundle adjustment method might be exploited by the selection of optimum system parameters to build accurate 3D skin lesion surface models (Table 1). In the tests, two different types of lesions are examined (elevated and flat). A sample of elevated skin lesion and its 3D model reconstruction is shown in Fig. 4.

For the tests each set of seven surface points on two types of lesion (elevated and flat), three check points and four control points located on the reference ring are used in association with the bundle adjustment algorithm. The lesion points can be selected from among other physical details (e.g. pigment dot, natural skin details, etc.) identified on the lesion (Fig.
5), and which are also visible on both stereo image pairs. The image pixel coordinates of the object points in both sets of two different camera positions $\left(\mathrm{S}_{1}, \mathrm{~S}_{2}\right)$ are measured by the image screening utility manually, and then used as inputs to the bundle adjustment utility. In this expe-riment the bundle adjustment algorithm is used in a close-range domain, as compared to industrial appli-cations, and hence any measurement error on control points coordinates are be more effective.

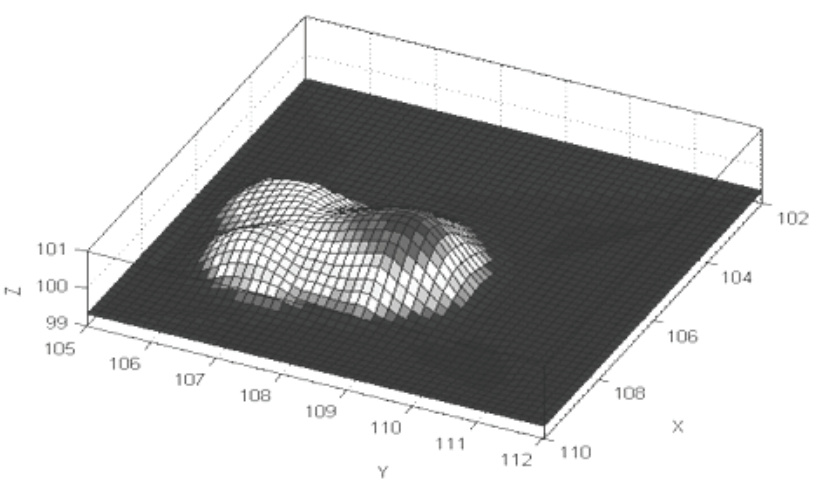

Fig. 4. An elevated lesion whose height (Z coordinates) are calculated by using photogrammetric bundle adjustment technique with the low base-toheight ratio: $B / H=0.2$. ( $3 D$ surface representation is shown). 


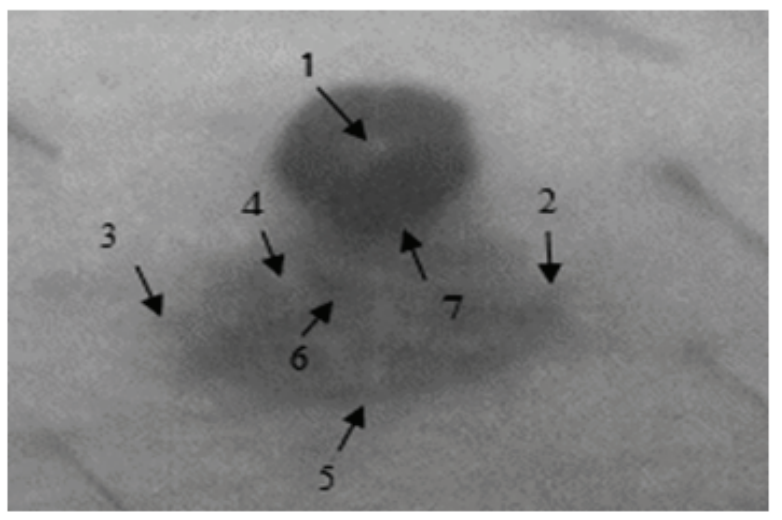

Fig. 5. The lesion points (pointed by arrows) are selected provided that they are clearly visible on both stereo image pairs and each selected point image coordinates with its corresponding ones are to be measured on both images.

In our experiments all image measurements on a skin lesion is made by using a low-cost digital camera by taking two sequential images (Fig. 1) targeting the same measurement area (including control and test points) from different point of views that called perspective centers Xs, Ys,Zs (Table 1). The focal length of the system remained constant during the image data collections, hence the camera should not have any "auto focus/zoom" facility.

On the reference ring (Fig. 2) the Cartesian $(\mathrm{X}, \mathrm{Y}, \mathrm{Z})$ coordinates of the control points and check points have been measured by using a digital gauge at approximately 10 micron accuracy. The accuracy of the calculated values for the check points yielded by bundle adjustment results corresponds to the accuracy of a reconstructed surface model of a skin lesion. In further stages of progressive 3D analysis of a lesion in a specific time period (e.g., to observe lesion growth, displacement, etc.), each set of model coordinates may compared to previous ones to identify the differences. It would be easier to compare model features (e.g., distances, volumes) rather than the coordinates because it will be difficult to position the reference ring in each time of measurement on its exact original location. The other issue is pin-point identification of control points' or test points' centroids to sub-pixel accuracy due to insufficient camera resolution (Fig. 6) and fixed focal length (non-auto focus) characteristic of the camera.
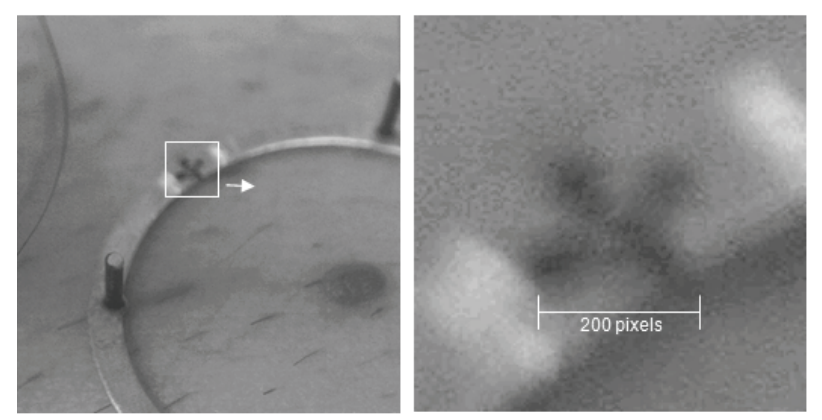

Fig. 6. By using relatively low resolution images of low-cost (non-metrological) cameras, it is difficult to identify pinpoint location of central pixel (centroid) of each control point marked as "+" (left image). The image blurring is also originated from non-focus characteristics of the camera due to fixed focal length requirement in bundle adjustment algorithm (right image)

By using relatively low resolution images taken by a low-cost (non-metrological) and non-focus style cameras, in some cases it may be difficult to pinpoint the location of the central pixel (centroid) on each control point. Incorrect location of a centroid pixel with \pm 1 pixel displacement may cause up to a few $\mathrm{mm}$ errors in 3D lesion coordinates. Fortunately these types of coordinate error can be automatically compensated for by the robust characteristics of the bundle adjustment method, but for some additional errors it may be more effectively applied to the final results, such as inaccurate focal length (less than 4 digit behind the decimal point) or CCD sensor matrix errors in a non-metrological camera. In our experiments, the results of the bundle adjustment calculations are matched with 3 check points (Fig. 2) forming a triangular area which help to estimate the errors of the lesion model coordinates $(X, Y, Z)$ for points $1-7$. Then by using $2 D$ interpolation method over the accurate triangular area, it is possible to correct lesion model coordinates with up to 10 micron accuracy (Table 1). The iteration procedure is followed by which the reverse solution of collinearity equation is made until a maximum accuracy is reached. By this procedure the software algorithm tries to converge to the optimum values by iteration where the number of iterations depend on the initial values selected arbitrarily. The closer the initial values are to the results, the smaller the number of iterations. 
Table 1. The results of $3 D$ model coordinates $(X, Y, Z)$ for four types of lesions at different elevations are yielded by bundle adjustment algorithm iterations. The further corrections to $Z$ coordinates $\left(\Delta_{i}\right)$ are calculated by interpolation over the Check points $(X, Y, Z)_{a, b, c}$ coordinate values (here the effect of point $C$ can be neglected). In the table, lesion model coordinates (points 1-7) and Check point coordinates ( $a, b$ and $c$ ) are shown for both lesions. To bring planimetric corrections to lesion points $\left(\Delta X_{i}, \Delta Y_{i}\right)$, simply the average of all 3 check points $(\Delta X, \Delta Y)_{a, b, c}$ are taken into account for an approximate results which may fall into a tolerance level. Here the planimetric $\left(\Delta X_{i}, \Delta Y_{i}\right)$ errors are less important than $\Delta Z_{i}$ elevation errors, since $\left(X_{i}, Y_{i}\right)$ can also be simply calculated by an interpolation on a single image by using check points $(X, Y)_{a, b, c}$ that are already known, disregarding the tilts of image. As only comparative lesion points $\left(\Delta X_{i}, \Delta Y_{i}\right)$ displacements would be counted. We have to note that if all points' calculated $X, Y$ coordinates have almost equal (or radial) displacements, then local lesion coordinates are not too much affected by these relatively large $X$, $Y$ planimetric displacements (in lesion $C$ and $D$ cases where radial and rotational displacements of all points exist respectively).

\begin{tabular}{|c|c|c|c|c|c|c|c|c|}
\hline \multicolumn{5}{|c|}{ LESION A - (MM) } & \multicolumn{4}{|c|}{ LESION B - (MM) } \\
\hline $\begin{array}{c}\text { Point } \\
\text { No. }\end{array}$ & $\mathrm{X}$ & $\mathrm{Y}$ & $\mathrm{Z}$ & $\begin{array}{c}\Delta \mathrm{X} \Delta \mathrm{Y} \Delta \mathrm{Z} \\
\text { corrections }(\mathrm{mm})\end{array}$ & $\mathrm{X}$ & Y & $\mathrm{Z}$ & $\begin{array}{c}\Delta \mathrm{X} \Delta \mathrm{Y} \Delta \mathrm{Z} \\
\text { corrections (mm) }\end{array}$ \\
\hline 1 & 105.32 & 106.26 & 99.33 & $-0.510 .43-0.37$ & 106.50 & 108.71 & 103.24 & $-0.04-1.03-0.16$ \\
\hline 2 & 105.75 & 106.10 & 100.11 & $\begin{array}{lll}-0.51 & 0.43-0.36\end{array}$ & 105.65 & 108.45 & 103.42 & $-0.04-1.03-0.15$ \\
\hline 3 & 105.16 & 107.48 & 99.45 & $\begin{array}{lll}-0.51 & 0.43 & -0.40\end{array}$ & 106.54 & 108.44 & 103.18 & $-0.04-1.03-0.15$ \\
\hline 4 & 105.84 & 106.49 & 100.26 & $\begin{array}{lll}-0.51 & 0.43-0.37\end{array}$ & 107.27 & 107.09 & 102.94 & $-0.04-1.03-0.10$ \\
\hline 5 & 107.01 & 107.10 & 100.33 & $\begin{array}{lll}-0.51 & 0.43 & -0.39\end{array}$ & 106.81 & 107.34 & 102.99 & $-0.04-1.03-0.11$ \\
\hline 6 & 107.75 & 106.52 & 100.32 & $\begin{array}{lll}-0.51 & 0.43-0.37\end{array}$ & 105.85 & 108.04 & 103.36 & $-0.04-1.03-0.14$ \\
\hline 7 & 106.83 & 108.74 & 100.95 & $\begin{array}{lll}-0.51 & 0.43 & -0.44\end{array}$ & 108.14 & 107.75 & 103.02 & $-0.04-1.03-0.12$ \\
\hline $\mathrm{a}$ & 109.24 & 116.69 & 105.11 & $-0.24-0.39-0.68$ & 110.91 & 118.59 & 104.91 & $-1.91-2.29-0.47$ \\
\hline b & 107.27 & 97.97 & 103.13 & $-0.541 .05-0.12$ & 105.85 & 98.48 & 102.79 & 0.880 .540 .22 \\
\hline $\mathrm{c}$ & 97.96 & 107.46 & 103.86 & -0.750 .640 .32 & 96.10 & 109.46 & 104.27 & $0.92-1.35-0.09$ \\
\hline \multicolumn{4}{|c|}{ LESION C - (MM) } & & \multicolumn{4}{|c|}{ LESION D - (MM) } \\
\hline 1 & 99.95 & 112.96 & 99.99 & $0.11 .7-1.3$ & 103.50 & 111.39 & 100.06 & $0.3-0.20 .2$ \\
\hline 2 & 101.02 & 113.20 & 100.67 & $0.051 .7-1.3$ & 105.69 & 109.88 & 100.89 & $\begin{array}{lll}0.3 & -0.2 & 0.2\end{array}$ \\
\hline 3 & 103.31 & 114.24 & 99.20 & $-0.21 .7-1.4$ & 101.47 & 108.95 & 100.77 & $0.3-0.20 .1$ \\
\hline 4 & 99.15 & 111.94 & 100.39 & $0.11 .7-1.3$ & 102.59 & 109.70 & 100.79 & $\begin{array}{llll}0.3 & -0.2 & 0.2\end{array}$ \\
\hline 5 & 101.25 & 115.00 & 99.43 & $0.051 .7-1.4$ & 103.68 & 108.46 & 99.39 & $\begin{array}{llll}0.3 & -0.2 & 0.1\end{array}$ \\
\hline 6 & 102.26 & 112.30 & 99.20 & $-0.11 .7-1.3$ & 103.23 & 109.41 & 100.88 & $\begin{array}{llll}0.3 & -0.2 & 0.2\end{array}$ \\
\hline 7 & 102.50 & 113.96 & 99.97 & $-0.11 .7-1.4$ & 103.99 & 110.56 & 99.94 & $0.3-0.20 .2$ \\
\hline $\mathrm{a}$ & 94.52 & 123.75 & 106.36 & $14.4-7.451 .93$ & 101.32 & 120.49 & 103.69 & $7.68-4.190 .73$ \\
\hline $\mathrm{b}$ & 109.23 & 106.30 & 103.88 & $-2.5-7.280 .86$ & 108.25 & 103.23 & 104.77 & $-1.52-4.21-1.76$ \\
\hline $\mathrm{c}$ & 92.61 & 105.66 & 105.12 & 4.52 .440 .94 & 94.47 & 107.43 & 105.33 & $2.730 .67-1.15$ \\
\hline
\end{tabular}

\section{DISCUSSION}

Before obtaining the stereo image acquisition the major camera interior parameters have to be calculated (focal length, CCD size, etc.). The focal length (principal distance) should be calculated $(\mathrm{f}=8.883 \mathrm{~mm}$ ) due to modifications of the camera lens system to enable the camera to take close-range images. This procedure was carried out by using an optic lab utility and geometric principles (Orun, 1996) which is described by Eq. 6 . The previous experiments have shown that (Orun and Natarajan, 1994) the best geometric configuration for accurate results of $\mathrm{Z}$ coordinates are established by ratio of base/height $=1$, where "base" refers to distance between two cameras' perspective centers and "height" is distance between the camera and skin surface. It has been proven that (Orun,1990) the optimum $\mathrm{B} / \mathrm{H}$ ratio for $\mathrm{Z}$ coordinates may degrade the planimetric accuracy of $X, Y$ coordinates. Hence in our work the height accuracy ( $\mathrm{Z}$ coordinates) are given a priority over the planimetric accuracy. This is because a single camera image (positioned vertically to skin surface) would be sufficient to calculate $X, Y$ coordinates of a lesion accurately by using basic perspective geometry principles such as ;

$$
X_{m m} \vee Y_{m m}=\frac{H_{m m} \cdot\left(\text { CCDpixelsize }_{m m}\right) \cdot\left(x \vee \text { y_imagecoordinate }_{\text {pixel }}\right)}{f_{m m}}
$$

where: $\mathrm{X}$ or $\mathrm{Y}$ are Cartesian coordinates in planimetric object domain, and $\mathrm{x}$ or $\mathrm{y}$ are their corresponding 
image coordinates, $\mathrm{H}$ is the distance between camera and skin surface, $f$ is the principal distance (or focal length if camera objective focuses on $\infty$ ), CCDpixelsize is the size of single CCD unit of camera (in $\mathrm{mm}$ ) and $\mathrm{x}$ or $\mathrm{y}$ image coordinates in pixel unit. According to the results shown in Table 1, the errors on the X,Y,Z coordinates of two lesion types can be easily calculated by using check points located on the reference ring (Fig. 2). Each check point was precisely measured by a digital gauge at 10 micron accuracy. The horizontal planimetric errors on $\mathrm{X}, \mathrm{Y}$ coordinates vary between $0.24 \mathrm{~mm}$ and $2.29 \mathrm{~mm}$ for the average distance $(15 \mathrm{~mm})$ between the check points whose locations surround the target lesion. If the maximum horizontal planimetric errors between the check points are distributed over the lesion points coordinates, the max horizontal error for the lesion points corresponds to $0.6 \mathrm{~mm}$. This error may be neglected since bundle adjustment method results have distance-preserving characteristics being effective on the planimetric object domain (distances between the lesion points). Normally even a single pixel measurement error on a single image may result a few millimeters $\mathrm{Z}$ coordinate errors on lesion points (Fig. 5) and this may increase if both image have the same symmetric pixel errors. But fortunately bundle adjustment method also has a unique self-compensation characteristics which applies corrections automatically to the lesion points coordinates. The display of calculated lesion points coordinates for 3D model is shown in Fig. 7.

The techniques introduced here can be used with any set of suitable camera equipment and may be easily adapted to any sensor system which has a basic perspective geometrical characteristic (e.g., microscope, etc.). This would widen its application areas in a broad range and its low-cost characteristics may also strengthen its market potential. The system has compact and portable characteristics, hence it may be used for the legal purposes for measuring or tracking any medical condition of a skin disorder or skin surface blemish after an injury (e.g., for insurance companies, Medicare reimbursement, etc.). The stereo image pair can be transmitted over the internet to clinical server for a periodical self assessment of the suspicious lesions. The repetition of same measurements will statistically give an average value of a lesion growth even though the measurement accuracy is not very high.

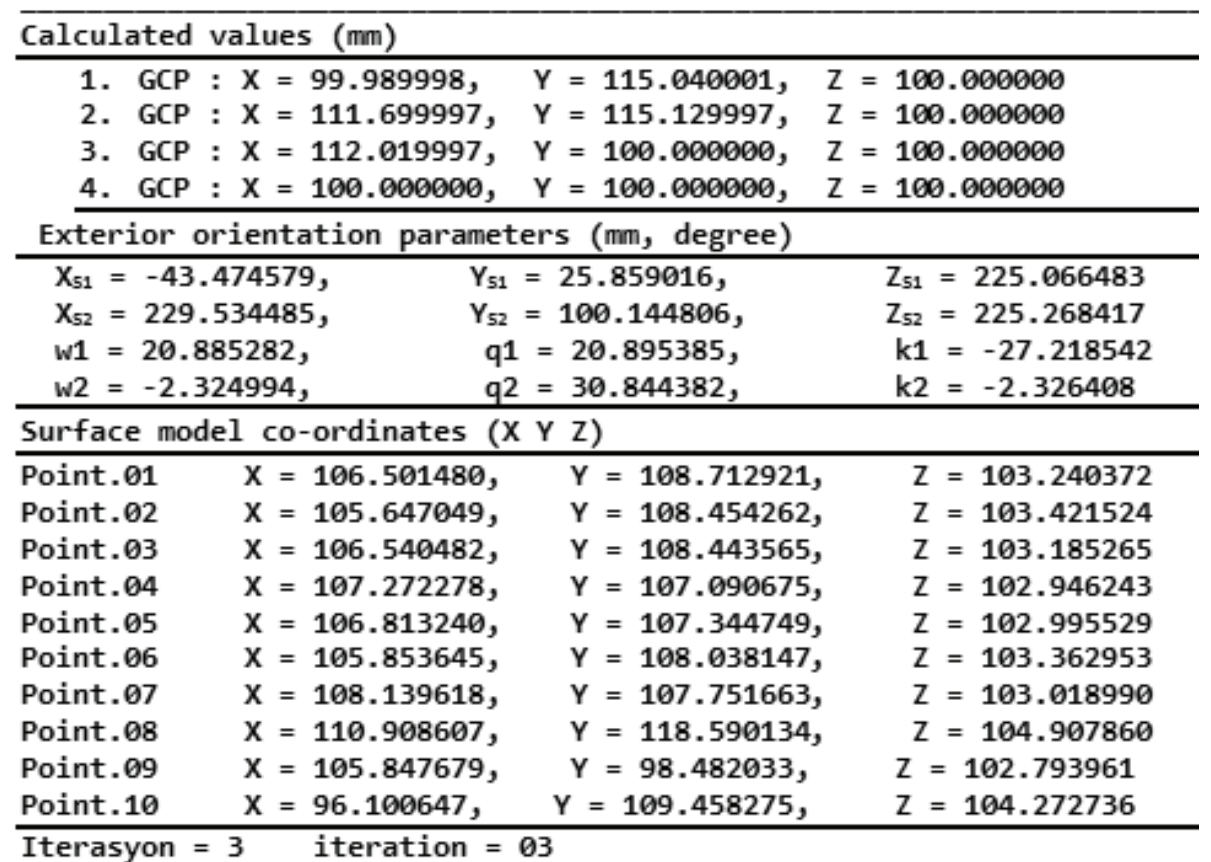

Fig. 7. The display of the Bundle adjustment algorithm results by which $3 D$ lesion model coordinates (in mm) are calculated after 3 iterations, which includes 7 lesion points (1-7) and 3 check points (8-10). Using this method, the lesion growth progress may also be observed by comparison between the set of coordinates sequentially obtained in a specific time period. Exterior orientation parameters include positions $\left(X s_{1}, X s_{2}\right)$ and tilts $(\omega, \varphi, \kappa)$ of a single digital Camera at two stationary locations. 


\section{REFERENCES}

Alvarez O, Windelken M, Markowitz L, Comfort CL, Waltrose L (2006). Wounds measured from digital photographs using photo-digital planimetry software: validation and rater reliability. $19^{\text {th }}$ Annual Symposium on Advanced Wound Care. USA, Texas 2006.

Choi JW, Lee JY, Oh T, Kwon SM, Yang, SJ, Koh KS (2013). Frontal soft tissue analysis using a 3D camera following two-jaw rotational orthognathic surgery in skeletal class III patients. Journal of Cranio-MaxilloFacial Surgery. 1-7.

Claridge E, Orun AB (2002). Modeling of edge profiles in pigmented skin lesions", (MIUA) Medical Image Understanding and Analysis, University of Portsmouth, July 2002.

Claridge E, Orun AB (2003). Characterizing pattern asymmetry in pigmented skin lesions. (MIUA) Medical Image Understanding and Analysis, Sheffield, July 2003.

Garding J (1993). Direct estimation of shape from texture. IEEE Trans. Pattern Analysis and Machine Intelligence 15:11.

Goellner M, Schmitt J, Karl M, Wichmann M, Holst S (2010). Photogrammetric measurement of initial tooth displacement under tensile force, Medical Engineering \& Physics 32:883-8.

Gorpas D, Politopoulos K, Yova D (2007). A binocular machine vision system for three-dimensional surface measurement of small objects, Computerized Medical Imaging and Graphics 31:625-37.

Granshaw SI (1980). Bundle adjustment methods in engineering photogrammetry. Photogrammetric Record 10: 181-207.

Hiraoglu M (1992). Characterisation and calibration of cameras for machine vision metrology. $\mathrm{PhD}$ Thesis, Rensselaer Polytechnic Institute. Dept. of Electrical, Computer and Systems Eng 1992: May.
Jaspers S, Hopermann H, Sauermann G, Hoppe U, Lunderstadt R, Ennen J (1999). Rapid in vivo measurement of the topography of human skin by active image triangulation using a digital micro mirror device. Skin Research and Technology 5:195-207.

Khalil AM (2011). Two dimensional displacement measurement using static close range photogrammetry and a single fixed camera. Alexandria Engineering Journal 50:219-27.

Luhmann T (2009). Precision potential of photogrammetric 6 DOF pose estimation with a single camera. ISPRS Journal of Photogrammetry and Remote Sensing 64: 275-84.

Moffitt FH, Mikhail EM (1980). Photogrammetry. Harper \& Row Publisher Inc. New York, USA.

Orun AB (1990). SPOT satellite imagery for topographic mapping. MPhil Thesis, Oxford Brookes University, Oxford UK, August 1990.

Orun AB, Natarajan K (1994). A modified bundle adjustment software for SPOT imagery and photography: A tradeoff. PE\&RS Journal, December 1994.

Orun AB (1996). Real-time photogrammetric vision system design and development. PhD Thesis. Yildiz Technical University, Faculty of Engineering, Istanbul, October 1996.

Orun AB, Alkis (2003). Material identification by surface reflection analysis in combination with bundle adjustment technique. Pattern Recognition Letters 24:1589-98.

Tominaga S (1991). Surface identification using the dichromatic reflection model. IEEE Trans Pattern Analysis \& Machine Intelligence, 13.

Witkin AP (1981). Recovering surface shape and orientation from texture. Artificial Intelligence 17:1.

Woodham RJ (1980). Photometric method for determining surface orientation from multiple images. Optical Engineering 19:1. 